\title{
Probing onset of strong localization and electron-electron interactions with the presence of direct insulator-quantum Hall transition
}

\author{
Shun-Tsung Lo ${ }^{1}$, Kuang Yao Chen ${ }^{1}$, T. L. Lin ${ }^{1}$, Li-Hung Lin ${ }^{2}$, Dong-Sheng \\ Luo $^{3}$, Y. Ochiai ${ }^{4}$, N. Aoki ${ }^{4}$, Yi-Ting Wang ${ }^{1}$, Zai Fong Peng ${ }^{2}$, Yiping \\ $\operatorname{Lin}^{3}$, J. C. Chen ${ }^{3}$, Sheng-Di Lin ${ }^{5}$, C. F. Huang ${ }^{6}$, and C.-T. Liang ${ }^{1, *}$ \\ ${ }^{1}$ Department of Physics, National Taiwan University, Taipei 106, Taiwan \\ ${ }^{2}$ Department of Applied Physics, National Chiayi University, Chiayi 600, Taiwan \\ ${ }^{3}$ Department of Physics, National Tsinghwa University, Hsinchu 300, Taiwan \\ ${ }^{4}$ Graduate School of Advanced Integration Science, \\ Chiba University, 1-33 Yayoi-cho, Inage-ku, Chiba 263-8522, Japan \\ ${ }^{5}$ Department of Electronics, National Chiao Tung University, Hsinchu 300, Taiwan and \\ ${ }^{6}$ National Measurement Laboratory, \\ Centre for Measurement Standards, \\ Industrial Technology Research Institute, Hsinchu 300, Taiwan
}

\begin{abstract}
We have performed low-temperature transport measurements on a disordered two-dimensional electron system (2DES). Features of the strong localization leading to the quantum Hall effect are observed after the 2DES undergoes a direct insulator-quantum Hall transition with increasing the perpendicular magnetic field. However, such a transition does not correspond to the onset of strong localization. The temperature dependences of the Hall resistivity and Hall conductivity reveal the importance of the electron-electron interaction effects to the observed transition in our study.

* E-mail addresses: ctliang@phys.ntu.edu.tw
\end{abstract}

PACS numbers: 


\section{INTRODUCTION}

When a strong magnetic field $B$ is applied perpendicular to the plane of a two-dimensional electron system (2DES), Landau quantization may cause the formation of Landau bands. It is now well established that Landau quantization can modify the electrical properties of a $2 \mathrm{D}$ system. With increasing $B$, usually Landau quantization may give rise to Shubnikov-de Haas $(\mathrm{SdH})$ oscillations with the amplitude [1 $\left[\begin{array}{ll}5 & \end{array}\right]$

$$
\triangle \rho_{x x}(B, T)=4 \rho_{0} \exp (-\pi / \mu B) D(B, T)
$$

in the longitudinal resistivity $\rho_{x x}$ before the appearance of the integer quantum Hall effect (IQHE) [1, 6] at a low temperature $T$. Here $\rho_{0}$ is expected to be the longitudinal resistivity $\rho_{x x}$ at $B=0$ while there may exsit deviations [7], $\mu$ is thequnatum mobility, and $D(B, T)=$ $2 \pi^{2} k_{B} m^{*} T / \hbar e B \sinh \left(2 \pi^{2} k_{B} m^{*} T / \hbar e B\right)$ with $m^{*}, k_{B}$, and $\hbar$ as the effective mass, Boltzmann constant, and reduced Plank constant. It is worth mentioning that the SdH theory is derived based on Landau quantization without considering the strong localization effects induced by the quantum interference. On the other hand, it is believed that both extended and localized states arising from such effects are key ingredients for describing the IQHE, in which the magnetic-field-induced transitions [8-12] are good examples of quantum phase transitions. Such transitions occur as the Fermi energy passes through the extended states of Landau bands. In the global phase diagram (GPD) [8] of the quantum Hall effect, all the magnetic-field-induced transitions are regarded equivalent though they are divided into two types, plateau-plateau (P-P) transitions and insulator-quantum Hall (I-QH) transitions [12].

There has been much interest in the IQHE at low magnetic fields [13-18]. A thorough understanding of the low-field IQHE should provide important information regarding the I-QH transition [8, 10, 11, 19]. In particular, whether a direct transition from the insulating regime (denoted by symbol 0 ) to a $\nu \geq 3 \mathrm{QH}$ state can occur is an interesting, fundamental yet unsettled issue in the field of two-dimensional (2D) physics [13-15, 20 23]. Experimental and theoretical evidence for such a direct phase transition has been reported [13 15,20 22]. On the other hand, it was argued by Huckestein [16] that the observed direct I-QH transition is not a real quantum phase transition, but a crossover from weak localization to strong localization regime in which Landau quantization becomes dominant. Within Huckestein's model, the onset of strong localization which causes the formation of a $\mathrm{QH}$ 
state should correspond to the direct I-QH transition. We note that in such a model, both weak localization and electron-electron interactions are considered as correction terms to the classical Drude conductivities. It is well known that electron-electron (e-e) interactions could play an important role in the metal-insulator transition (MIT) [24]. Moreover, in the seminal work of Dubi, Meir and Avishai [25], various transitions such as I-QH, MIT, and percolation transitions [26, 27] can be explained within a unifying model. Therefore, it is interesting to probe electron-electron interaction effects with the presence of I-QH transition. Moreover, the effect of Landau quantization and onset of strong localization are fundamental issues regarding the direct I-QH transition.

In this communication, we report magneto transport measurements on a disordered 2DES. With increasing $B$, the strength of the strong localization increases such that we can observe the well-developed $\mathrm{QH}$ state of $\nu=2$. However, the direct I-QH transition observed at $B \sim 2.29 \mathrm{~T}$ is not due to the onset of the strong localization because $\mathrm{SdH}$ formula is valid as $B<4.76 \mathrm{~T}$. The $T$-dependences of the Hall resistivity $\rho_{x y}$ and Hall conductivity $\sigma_{x y}$ show the importance of e-e interactions to such a transition in our study.

\section{EXPERIMENTAL DETAILS}

Sample LM4645, a delta-doped quantum well with additional modulation doping, is used in this study. The following layer sequence was grown on a semi-insulating GaAs (100) substrate: $500 \mathrm{~nm}$ GaAs, $80 \mathrm{~nm} \mathrm{Al}_{0.33} \mathrm{Ga}_{0.67} \mathrm{As}, 5 \mathrm{~nm}$ GaAs, Si delta-doping with a concentration of $3 \times 10^{11} \mathrm{~cm}^{-2}$, $15 \mathrm{~nm}$ GaAs, $20 \mathrm{~nm} \mathrm{Al} \mathrm{Al}_{0.33} \mathrm{Ga}_{0.67} \mathrm{As}, 40 \mathrm{~nm}$ Si-doped $\mathrm{Al}_{0.33} \mathrm{Ga}_{0.67} \mathrm{As}$

with a doping concentration of $10^{18} \mathrm{~cm}^{-3}$, finally $10 \mathrm{~nm}$ GaAs cap layer. Experiments were performed in a top-loading $\mathrm{He}^{3}$ cryostat equipped with a superconducting magnet. Four-terminal magneto resistivities were measured using standard ac phase-sensitive lock-in techniques. Magnetic field is applied perpendicular to the plane of the 2DES.

\section{RESULTS AND DISCUSSION}

Figure 1 shows longitudinal and Hall resistivities $\left(\rho_{x x}\right.$ and $\left.\rho_{x y}\right)$ as a function of magnetic field $B$ at various temperatures $T$. For $2.54 \mathrm{~T} \leq B \leq 4.76 \mathrm{~T}$, magneto-oscillations in $\rho_{x x}$ are observed. In order to further study these oscillations, we plot their amplitudes as a function 
of $1 / B$ as shown in Fig. 2. As shown in this figure, there is a good fit to Eq. 1, and thus these magneto-oscillations are ascribed to $\mathrm{SdH}$ oscillations. From the observed $\mathrm{SdH}$ oscillations, the carrier density of the 2 DES is measured to be $3.97 \times 10^{15} \mathrm{~m}^{-2}$. According to the fit shown in Fig. 2, the quantum mobility is estimated to be $\approx 0.19 \mathrm{~m}^{2} / \mathrm{Vs}$. Since the SdH theory is derived based on Landau quantization without considering strong localization effects, it is believed that high-field strong localization effects leading to the IQHE are not significant for $B \leq 4.76 \mathrm{~T}$. The resistance peak at around $6 \mathrm{~T}$ appears to move with increasing $B$. This movement cannot be described within the standard SdH theory and the measured amplitudes at $B=4.76 \mathrm{~T}$ can be affected by this movement. Hence the data point at $B=4.76 \mathrm{~T}$ in Fig. 2 are in open symbols whilst it can be fitted to Eq. (1).

The Hall slope at low $B$ increases with decreasing $T$, and we can see from Fig. 1 that the curves of $\rho_{x y}$ at $T=0.33 \mathrm{~K}$ and $T=1.242 \mathrm{~K}$ do not collapse into a single curve as $B$ is smaller than $4 \mathrm{~T}$. Such a change on the Hall slope does not result from a change in $n$ since $n$ determined from the $\mathrm{SdH}$ oscillations is $T$-independent over the whole measurement range. As will be described later, such $T$-dependent $\rho_{x y}$ can be ascribed to electron-electron interactions.

As shown in the inset to figure 1 , for $7.6 \mathrm{~T} \leq B \leq 10.6 \mathrm{~T}$, we can see a well-quantized $\nu=2$ Hall plateau with corresponding vanishing resistivity. Therefore the strong localization effect which gives rise to the formation of the quantum Hall state should occur with increasing $B$.

In order to further study the strong localization effect in our system, we follow the seminal work of Shahar [12] as described as follows. First, as shown in Fig. 3. we convert the measured $\rho_{x x}$ and $\rho_{x y}$ into $\sigma_{x x}$ and $\sigma_{x y}$ by matrix inversion

$$
\begin{aligned}
\sigma_{x x} & =\frac{\rho_{x x}}{\rho_{x x}^{2}+\rho_{x y}^{2}}, \\
\sigma_{x y} & =\frac{\rho_{x y}}{\rho_{x x}^{2}+\rho_{x y}^{2}} .
\end{aligned}
$$

Using the following equations, we then obtain the conductivity of the topmost Landau level by subtracting from the conductivity data the contribution of the lowest, full Landau level.

$$
\begin{gathered}
\sigma_{x x}^{t}=\sigma_{x x}, \\
\sigma_{x y}^{t}=\sigma_{x y}-\frac{2 e^{2}}{h} .
\end{gathered}
$$


Finally we convert $\sigma_{x x}^{t}$ and $\sigma_{x y}^{t}$ into the corresponding resisitivities for the topmost Landau levels $\rho_{x x}^{t}$ and $\rho_{x y}^{t}$. Such results are shown in Fig. 4. We can clearly see a clear crossing point in $\rho_{x x}^{t}$ at around $5.2 \mathrm{~T}$, which is denoted by a vertical dotted line. Such a $T$-independent point can be ascribed to the formation of the extended states under the existence of the localized states [12]. Since both extended and localized states are due to strong localization effects leading to the IQHE, such effects should become significant as $B \geq 5.2 \mathrm{~T}$ in our system. On the other hand, at $B<4.76 \mathrm{~T}$ the validity of $\mathrm{SdH}$ formula reveals that the strength of strong localization is weak. Therefore, the onset of the strong localization occurs as $B=4.76 \mathrm{~T} \sim 5.2 \mathrm{~T}$.

It has been pointed out that the strong localization occurs at the magnetic field $B \sim 1 / \mu$, near which the localization length changes quickly [16]. Just as mentioned in the above, the mobility $\mu=0.19 \mathrm{~m}^{2} / \mathrm{Vs}$ and thus the onset of the strong localization is expected as $B \sim 5.3 \mathrm{~T}$. Such a magnetic field is close to the estimation based on SdH formula and the crossing point in $\rho_{x x}^{t}$. We note that the temperature-independent point in $\rho_{x x}^{t}$ is close to the resistance quantum $\frac{h}{2 e^{2}}$ as expected for the topmost Landau level [12]. In addition, as shown in Fig. $4, \rho_{x y}^{t}$ does not deviate much from the expected value $\frac{h}{2 e^{2}}$ at the lowest temperature $T=0.33 \mathrm{~K}$ as $B<5.2 \mathrm{~T}$.

We can see from Fig. 1 that the 2DES behaves as an insulator as $B<B_{c} \equiv 2.29 \mathrm{~T}$ in the sense that $\rho_{x x}$ increases with decreasing $T$. The longitudinal resistivity $\rho_{x x}$ is almost independent of $T$ at $B_{c}$. Since there is no $\mathrm{QH}$ state of the lowest integer filling factor 1 or 2 near $B_{c}$, the 2DES undergoes a direct I-QH transition at $B_{c}[13,15]$. The filling factor $\nu$ is about 8 near $B_{c}$, so the observed transition is a 0-8 transition [15, 20]. If such a transition is due to the onset of the strong localizationc [16], $B_{c}$ should be within $B=4.76 T \sim 5.2 \mathrm{~T}$ as mentioned above. In our study, however, $B_{c}$ is at a much lower magnetic field $B=2.29 \mathrm{~T}$. Therefore, the observed direct I-QH transition is not due to the onset of strong localization.

It has been shown that by converting the measured $\rho_{x x}$ and $\rho_{x y}$ into longitudinal and transverse conductivities $\sigma_{x x}$ and $\sigma_{x y}$, one can provide further information on the I-QH transition [11, 28]. Figure 3 shows converted $\sigma_{x x}$ and $\sigma_{x y}$ as a function of $B$. We can see that $\sigma_{x y}$ is $T$-independent over a wide range of magnetic field $(0 \mathrm{~T} \leq B \leq 2.8 \mathrm{~T})$, spanning from the insulating region to the QH-like regime. On the other hand, as shown in the inset to Fig. 3, the Hall slope $R_{H}$ shows an approximately $\ln T$ dependence. The deviation from the linear fit through the full symbols can be ascribed to current heating. 
As the current is decreased from $20 \mathrm{nA}$ to $10 \mathrm{nA}$ (full circle in blue), we are able to restore the $\ln T$ dependence at low $T$ [23]. The observed $\ln T$ dependence of $R_{H}$ does not result from a change in $n$ since $n$ determined from the $\mathrm{SdH}$ oscillations is $T$-independent over the whole measurement range. Therefore, the observed $T$-indepndent $\sigma_{x y}$, together with the $\ln T$-dependent $\rho_{x y}$ can be ascribed to electron-electron interaction, and we note that the corrections resulting from such an interaction have been discussed in the literature [30]. Our experimental result therefore supports that the direct I-QH transition is not always due to the onset of strong localization when the e-e interaction is significant.

Interestingly, whilst there is a crossing field at $B_{c}$ in $\rho_{x x}$, there is no corresponding crossing point in $\sigma_{x x}$ [29]. The reason for this is that $\rho_{x y}$ shows logarithmic dependence on $T$. Therefore according to Eq. 2, there is no corresponding crossing point in $\sigma_{x x}$ even there exists a $T$-independent point in $\rho_{x x}$.

To further study the direct I-QH transition and onset of the strong localization leading to the IQHE, we have re-analyzed the data published in Ref. [23], where the studied sample is almost identical except a different delta-doping concentration of $5 \times 10^{11} \mathrm{~cm}^{-2}$. There also exsits a crossing point in $\rho_{x x}^{t}$ ast $B \sim 1 / \mu$, near which the onset of the strong localization is expected. The direct I-QH transition, however, appears at a much lower magnetic field $B<$ $1 /(2 \mu)$ and does not correspond to the onset of strong localization. Tilted-field measurements show that the sample studied in Ref. [23] is two-dimensional such that the direct I-QH transition and features of Landau quantization only depend on the perpendicular component of the applied $B$.

It has been reported in some case when $\rho_{x x}$ approaches zero and the strong localization effects may occur, the large resistance oscillations can be still well approximated by the conventional SdH formula [17, 18]. In this case, rising background resistance [17] needs to be introduced while such background resistance does not occur in our system. In our system, the amplitudes of the resistance oscillations are a lot smaller than the non-oscillating background as $B \leq 4.76 \mathrm{~T}$, under which the resistance minima are much bigger than zero.

Based on the tight-binding model, Nita, Aldea and Zittartz [31] have predicted that resistance oscillations can cover the I-QH transition. There exists also experimental evidence for this prediction [32]. We note that in this case, e-e interaction effects are not significant since $\rho_{x y}$ is nominally $T$-independent. It may be possible that the existence of e-e interactions may dictate the observation of SdH-like oscillations spanning from the insulating regime to 
the QH-like regime.

\section{CONCLUSION}

In conclusion, we have performed magneto transport measurements on a weaklydisordered 2DES. With increasing magnetic field, the 2DES undergoes a direct 0-8 I-QH transition at a crossing field $B_{c}$. For $B>B_{c}$, magneto-oscillations governed by conventional Shubnikov-de Haas theory are observed. Since strong localization effect is not considered in the $\mathrm{SdH}$ theory, our results explicitly demonstrate that the direct I-QH transition does not correspond to the onset of strong localization. The observed nominally $T$-independent $\sigma_{x y}$ spanning from the insulating regime to the $\mathrm{SdH}$ regime, together with the observed logarithmic $T$-dependent Hall slope demonstrate that electron-electron interactions, rather than the weak localization effects, are the dominant mechanism near the direct I-QH transition in our study.

Acknowledgment

This work was funded by the NSC, Taiwan. We would like to thank J.-Y. Wu and Y.-C. Su for experimental help.

[1] P. T. Coleridge, Semicond. Sci. Technol. 5 (1990) 961.

[2] P. T. Coleridge, R. Storner, and R. Fletcher, Phys. Rev. B 39 (1989) 1120.

[3] A.B. Fowler, F.F. Fang, W.E. Howard, P.J. Stiles, Phys. Rev. Lett. 16 (1966) 901.

[4] T. Ando, J. Phys. Soc. Jpn. 37 (1974) 279.

[5] A. Isihara, L. Smrcka, J. Phys. C 19 (1986) 6777.

[6] K. von Klitzing, G. Dorda, and M. Pepper, Phys. Rev. Lett. 45 (1980) 494.

[7] J. H. Chen, D. R. Hang, C. F. Huang, T. Y. Huang, J. Y. Lin, S. H. Lo, J. C. Hsiao, M. G. Lin, M. Y. Simmons, D. A. Ritchie, C.-T. Liang, J. Korean Phys. Soc. 50 (2007) 776.

[8] S. Kivelson, D. H. Lee, S. C. Zhang, Phys. Rev. B 46 (1992) 2223.

[9] Y. Huo, R. E. Hetsel, and R. N. Bhatt, Phys. Rev. Lett. 70 (1993) 481.

[10] H. W. Jiang, C. E. Johnson, K. L. Wang, S. T. Hannahs, Phys. Rev. Lett. 71 (1993) 1439

[11] R. J. F. Hughes, J. T. Nicholls, J. E. F. Frost, E. H. Linfield, M. Pepper, C. J. B. Ford, D. A. 
Ritchie, G. A. C. Jones, Eugene Kogan, Moshe Kaveh, J. Phys.: Condens. Matter 6 (1994) 4763.

[12] D. Shahar, D.C. Tsui, M. Shayegan, E. Shimshoni, S.L. Sondhi, Phys. Rev. Lett. 79 (1997) 479.

[13] D. Z. Liu, X. C. Xie, Q. Niu, Phys. Rev. Lett. 76 (1996) 975.

[14] D. N. Sheng, Z. Y. Weng, Phys. Rev. B 62 (2000) 15363.

[15] S.-H. Song, D. Shahar, D. C. Tsui, Y. H. Xie, and D. Monroe, Phys. Rev. Lett. 78 (1997) 2000.

[16] B. Huckestein, Phys. Rev. Lett. 84 (2000) 3141.

[17] D.R. Hang, C.F. Huang, Y.W. Zhang, H.D. Yeh, J.C. Hsiao, H. L. Pang, Solid State Commun. $141(2007) 17$.

[18] D.R. Hang, C.F. Huang, K.A. Cheng, Phys. Rev. B 80 (2009) 085312.

[19] G.-H. Kim, C.-T. Liang, C.F. Huang, J.T. Nicholls, D.A. Ritchie, P.S. Kim, C.H. Oh, J.R. Juang, Y. H. Chang, Phys. Rev. B 69 (2004) 073311.

[20] C. H. Lee, Y. H. Chang, Y. W. Suen, H. H. Lin, Phys. Rev. B 58 (1998) 10629.

[21] C. F. Huang, Y. H. Chang, C. H. Lee, H. T. Chou, H. D. Yeh, C.-T. Liang, Y. F. Chen, H. H. Lin, H. H. Cheng, G. J. Hwang, Phys. Rev. B 65 (2002) 045303.

[22] T.-Y. Huang, J. R. Juang, C. F. Huang, G.-H. Kim, C. P. Huang, C.-T. Liang, Y. H. Chang, Y. F. Chen, Y. Lee, D. A. Ritchie, Physica E 22 (2004) 240.

[23] K.Y. Chen, Y.H. Chang, C.-T. Liang, N. Aoki, Y. Ochiai, C.F. Huang, L.-H. Lin, K.A. Cheng, H.H. Cheng, H.H. Lin, J.-Y. Wu, S.-D. Lin, Journal of Physics: Condensed Matter 20 (2008) 295223

[24] V. Dobrosavljevic , E. Abrahams, E. Miranda, S. Chakravarty, Phys.Rev.Lett.79 (1997) 455.

[25] Y. Dubi, Y. Meir, Y. Avishai, Phys. Rev. Lett. 94 (2005) 156406.

[26] Y. Meir, Phys. Rev. Lett. 83 (1999) 3506.

[27] E. Shmishoni, A. Auerbach, and A. Kapitulnik, Phys. Rev.Lett. 80 (1998) 3352.

[28] Yu. G. Arapov, M.V. Yakunin, S. V. Gudina, G.I. Harus, V.N. Neverov, N.G. Shelushinina, S.M. Podgornyh, E.A. Uskova, B.N. Zvonkov, AIP conference proceedings 893 (2007) 647.

[29] T.-Y. Huang, C.-T. Liang, G.-H. Kim, C.F. Huang, C.-P. Huang, D.A. Ritchie, Physica E 42 (2010) 1142 .

[30] M.Y. Simmons, A.R. Hamilton, M. Pepper, E. H. Linfield, P.D. Rose, D. A. Ritchie, Phys. 
Rev. Lett. 84 (2000) 2489 and references therein.

[31] M. Nita, A. Aldea, J. Zittartz, Phys. Rev. B 62 (2000) 15367.

[32] G. Bohra, E. Comfort, T.-Y. Lin, M.-G. Kang, G. Strasser, J.P. Bird, C.F. Huang, L.-H. Lin, C.-T. Liang (unpublished).

Figure 1 Longitudinal resistivity $\rho_{x x}$ measurements as a function of magnetic field $B$ at various temperatures $T$. The Hall resistivity measurements at the lowest and highest $T$ so as to highlight its weak $T$ dependence. The inset shows both $\rho_{x x}$ and $\rho_{x y}$ measurements in the high-field regime at the lowest temperature $T=0.33 \mathrm{~K}$.

Figure $2 \Delta \rho_{x x} / D(B, T)$ as a function of $1 / B$ at various temperatures $T$ where $\Delta \rho_{x x}$ represents the amplitude of $\mathrm{SdH}$ oscillations. The solid curve corresponds to a fit to Eq. (1)

Figure 3 Converted (a) $\sigma_{x x}(B)$ and $(\mathrm{b}) \sigma_{x y}(B)$ at various temperatures $T$ ranging from $T=0.33 \mathrm{~K}$ to $T=1.242 \mathrm{~K}$. Inset: semilogarithmic plot of Hall slope $R_{H}$ as a function of $\ln T$. The linear fit to the full symbols is discussed in the text.

Figure 4 Converted $\rho_{x x}^{t}$ as a function of $B$ at various temperatures $T$ ranging from $T=$ $0.33 \mathrm{~K}$ to $T=1.242 \mathrm{~K} . \rho_{x y}^{t}$ is at $T=0.33 \mathrm{~K}$. The vertical dotted line denotes the magnetic field where $\rho_{x x}^{t}$ is $T$-independent. 


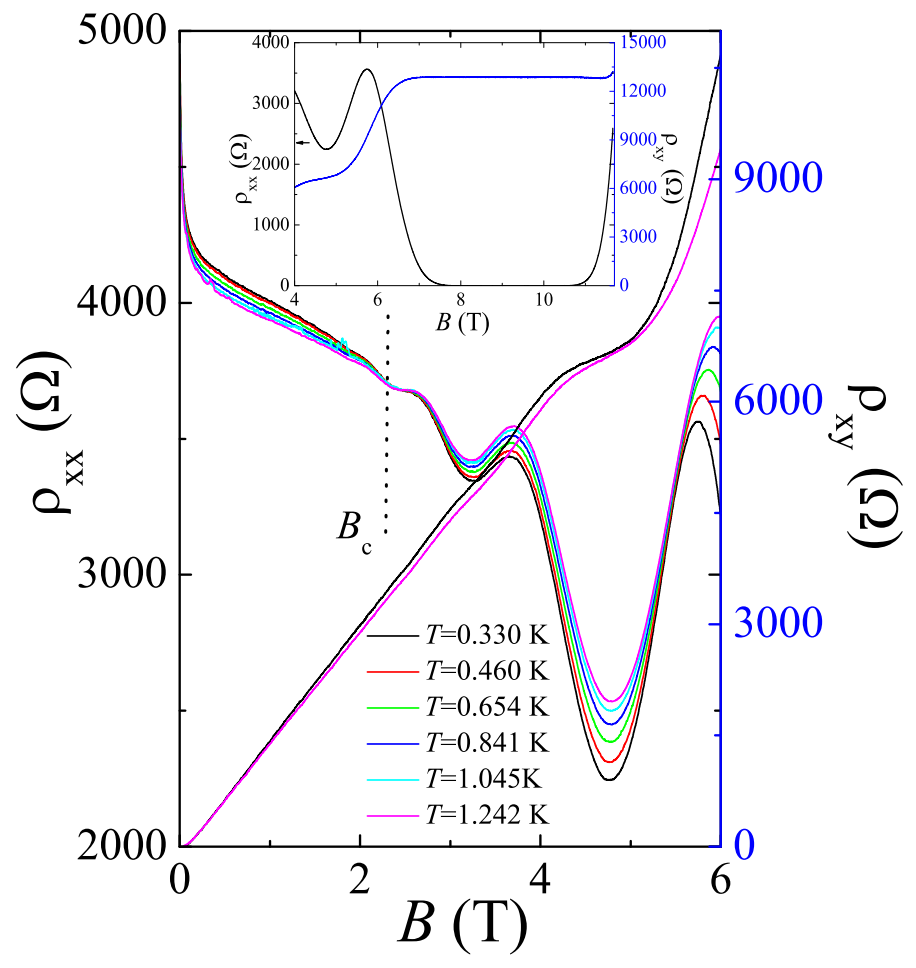




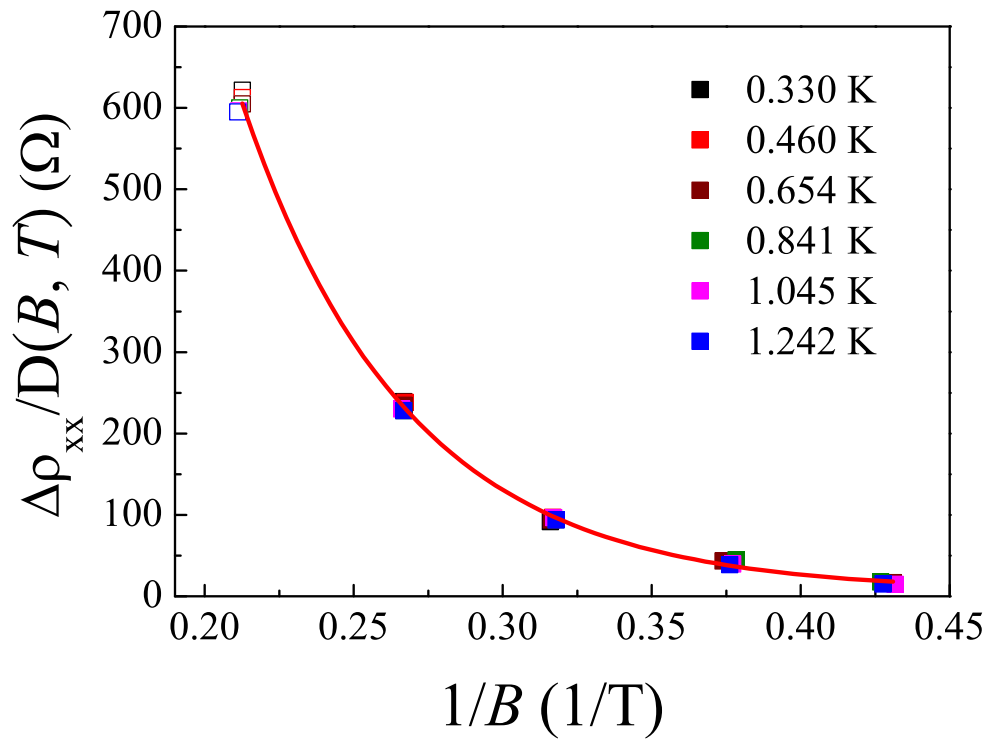




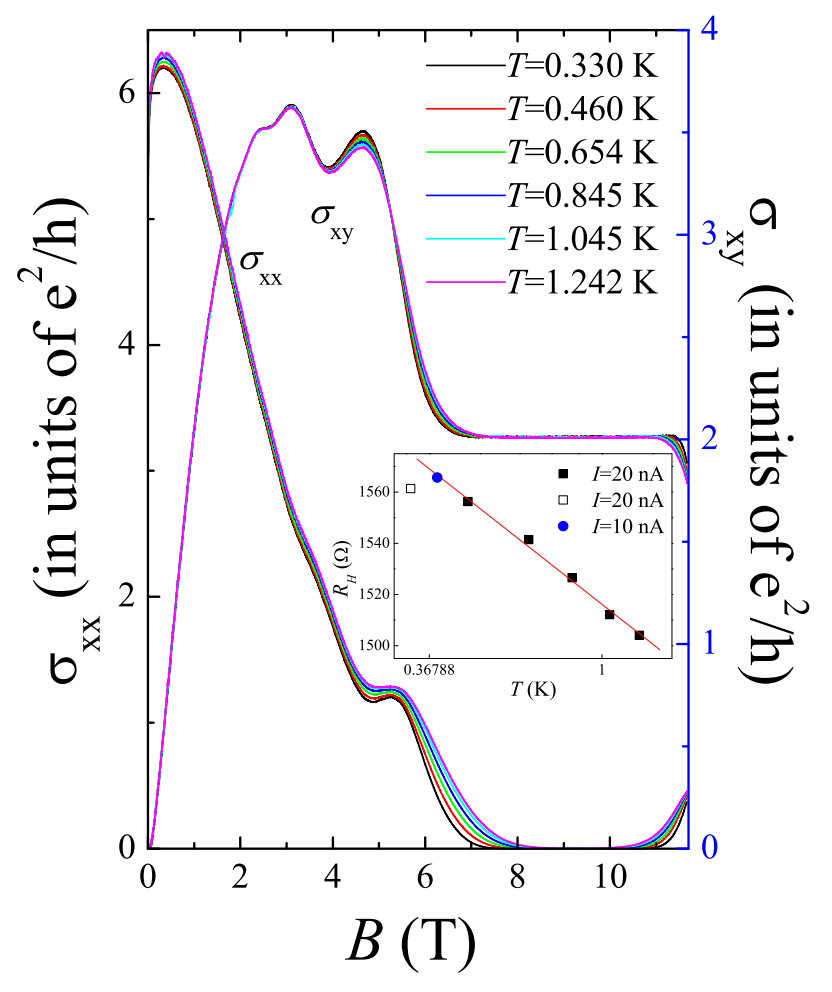




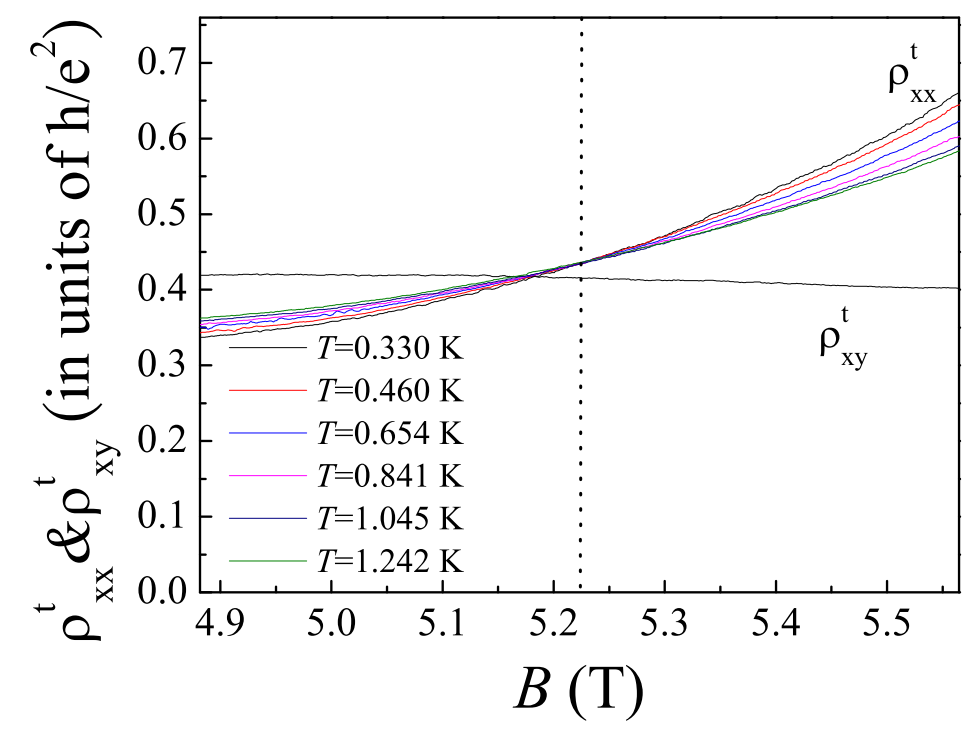

